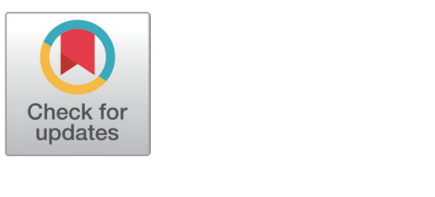

Received: Aug 17, 2020

Revised: Oct 8, 2020

Accepted: Oct 28, 2020

*Corresponding author Gaurishankar Manandhar Central Department of Biotechnology, Tribhuvan University, Kirtipur, Kathmandu 44618, Nepal. Tel: +977-01-4336221

E-mail: gaurishankar.manandhar@ gmail.com

Copyright $\odot 2021$ Korean Society of Animal Sciences and Technology.

This is an Open Access article distributed under the terms of the Creative Commons Attribution Non-Commercial License (http:// creativecommons.org/licenses/by$\mathrm{nc} / 4.0 /$ ) which permits unrestricted non-commercial use, distribution, and reproduction in any medium, provided the original work is properly cited.

ORCID

Sabina Bhandari

https://orcid.org/0000-0002-6913-9268 Jayaswori Sharma

https://orcid.org/0000-0002-5123-7396 Sarbesh Rizal

https://orcid.org/0000-0002-1520-7325 Young-Joo Yi

https://orcid.org/0000-0002-7167-5123

Gaurishankar Manandhar

https://orcid.org/0000-0002-8145-9475

Competing interests

No potential conflict of interest relevant

\section{Artemisia vulgaris extract causes precocious acrosome reaction and viability loss but low rate of membrane damage in mouse spermatozoa}

\author{
Sabina Bhandari ${ }^{1}$, Jayaswori Sharma ${ }^{1}$, Sarbesh Rizal ${ }^{1}$, Young-Joo Yi $^{2}$ and \\ Gaurishankar Manandhar ${ }^{1 *}$ \\ ${ }^{1}$ Central Department of Biotechnology, Tribhuvan University, Kirtipur, Kathmandu 44618, Nepal \\ ${ }^{2}$ Department of Agricultural Education, College of Education, Sunchon National University, Suncheon \\ 57922, Korea
}

\begin{abstract}
Several herbs including Artemisia are known to possess conceptive property. In the present study, mouse spermatozoa were incubated with ethanol extract of Artemisia vulgaris leaves. The effect of extract on acrosome exocytosis was studied by labeling spermatozoa with fluorescein isothiocyanate (FITC) peanut agglutinin and by staining with Coomassie blue. Viability and membrane integrity were studied by Trypan-blue staining and hypo-osmotic swelling test. Artemisia extract at very low concentration caused precocious acrosome reaction and loss of sperm viability. Acrosome reaction increased remarkably from $22.63 \%$ to $88.42 \%$ with increasing extract concentration from 0 to $2,000 \mu \mathrm{g} / \mathrm{mL}$. However, the viability loss of spermatozoa was increased from $11.71 \%$ in control to $63.73 \%$ in samples treated, evaluated by Trypan-blue staining method. Membrane damage caused by the extract, evaluated by hypo-osmotic swelling test was even low, ranging from $2.27 \%$ to only $24.23 \%$. These results indicate that Artemisia extract might block fertilization by causing precocious acrosome exocytosis in spermatozoa. A direct contraceptive effect was tested by injecting the plant extract into the vagina of female mice and then allowing them to mate with normal males. The treated female mice delivered significantly fewer litters in comparison to the control.
\end{abstract}

Keywords: Artemisia, Spermatozoa, Acrosome, Viability, Membrane damage

\section{INTRODUCTION}

Presently available allopathic contraceptives are costly and cause multiples of problems like hormonal imbalance and various other side effects [1]. An ideal alternative to allopathic contraceptives could be herbal extracts which could be applied topically when required and which would directly affect gamete function without causing any hormonal imbalance. Several medicinal plants have been investigated for various biochemical active components possessing contraceptive properties [2]. Owing to low side 
to this article was reported.

Funding sources

Young-Joo Yi was supported by the Basic

Science Research Program through the

National Research Foundation of Korea

(NRF) funded by the Ministry of Education

(NRF-2013R1A6A3A04063769).

\section{Acknowledgements}

We thanks Prof. Dr. Krishna Das Manandhar,

Head of Department, Central Department

of Biotechnology, Tribhuvan University for

providing lab facility and PhD scholar Mr.

Ramanuj Rauniyar for providing various

helps during this study. Fluorescein

isothiocyanate peanut agglutinin (FPNA)

used in the present work was generously

donated by Prof. P. Sutovsky, University of

Missouri, USA.

\section{Availability of data and material Upon reasonable request, the datasets of this study can be available from the} corresponding author.

\section{Authors' contributions}

Conceptualization: Yi YJ, Manandhar G.

Data curation: Bhandari S, Sharma J.

Formal analysis: Yi YJ.

Methodology: Bhandari S, Sharma J, Rizal S.

Validation: Bhandari S, Manandhar G.

Investigation: Bhandari S.

Writing - original draft: Bhandari S.

Writing - review \& editing: Manandhar G.

Ethics approval and consent to participate This research has been approved by the Institutional Ethic Committee of the Central Department of Biotechnology, Tribhuvan University, Nepal (CDBT/TU 084/2019). effects, they have gained considerable attention in recent years.

Plants with anti-fertility effects include Rubica cardiofolia, Artemisia vulgaris, Piper nigrum, Juniperus phoenica, Cuscuta reflexa, Tinospora cardiofolia, Acyranthes aspera etc. [2]. Ethanol extract of Tinospora cordifolia was found to be effective in producing reversible sterility, weight reduction of the reproductive organs like testis, epididymis, seminal vesicle and ventral prostate of treated male rats. The extract was found to cause significant reduction in the sperm density, motility and fertility in mice [3]. The induced infertility was completely reversed after withdrawal of the treatment. Alcoholic extract of Iranian neem (Azadirachta indica) seeds significantly reduced the motility and fertlity of mouse epididymal spermatozoa thus suggesting that the extract could be as a potential anti-fertility agent [4].

Artemisia is a diverse and large genus of plants, belonging to the family Asteraceae. Genus Artemisia comprises over 500 species of important medicinal plants, mainly found around Asia, Europe and North America. They are currently the subject of numerous phytochemical research and are found to possess varieties of essential oils [5]. A. vulgaris is one of the important perennial and aromatic medicinal herbs, 1-2 m tall, growing at the sides of paths and tracks and margins of cleared forests. It is known as mugwort, sailor's tobacco, common wormwood, wild wormwood, felon herb, old Uncle Henry, St. John's plant etc. and also known as 'pati' or 'titepati (bitter-leaf plant)' in Nepal. Leaves are 5-20 cm long, pinnate, sessile, alternate, simple and the margins are often rolled back. The plant possess a broad spectrum of therapeutic properties like antitumor antispasmodic, anti-steroidogenic, anti-fertility, antihypertensive, muscle relaxant, antiviral, antibacterial, antioxidant, cholinergic, diuretic etc. [6,7]. It is an important medicinal plant due to the presence of anti-malarial drug artemisinin [8], sesquiterpenes (derivative of artemisinin) and several essential oils [9]. Phytochemical screening of leaf extract of $A$. vulgaris, showed the presence of high amount of alkaloids, flavonoids, and terpenoids, steroids and tannin [10].

In addition to various therapeutic uses, $A$. vulgaris leaves extract shows anti-implantation, estrogenic activities in rats [11] and anti-fertility effect on Callosobruchus chinensis (weevil) through the effect on hatchability of pupae [12]. Alcoholic extract of $A$. vulgaris was found to induce an irregular estrous cycle and anti-implantation effect in female albino rats [13]. The herbal extract when fed to pregnant female mice as a dietary supplement in high dose did not cause gross malformations in pups, proving its non-toxic nature $[13,14]$. In the present work, we have shown that $A$. vulgaris extract causes high rate of precocious acrosome reaction in mouse spermatozoa, but lesser extent of membrane damage or viability loss. Intravaginal application of the extract reduces fertility significantly.

\section{MATERIALS AND METHODS}

\section{Reagents and chemicals}

All reagents and chemicals were purchased from Emplura-Sigma Aldrich, Bangalore, India unless otherwise mentioned.

\section{Collection of plant material and extract preparation}

Leaves were collected from plants before onset of flowering, growing wild in Kirtipur campus of Tribhuvan University, Kathmandu. They were washed by running tap water to remove the surface contamination. The leaves were dried in shade overnight followed by oven drying at $60^{\circ} \mathrm{C}$. Dry leaves were grinded to fine powder using a mechanical blender (Phillips, India). $100 \mathrm{~g}$ of powder was mixed with $80 \%$ ethanol in 1:3 ratio in a dark bottle. The mixture was kept in shaker for three overnights. The extract was filtered through Whatman No. 1 filter paper (GE Healthcare, Mumbai, India). The filtered extract was then preserved in an airtight dark bottle in a refrigerator for further studies. 


\section{Mouse husbandry and handling}

Male and female Swiss albino mice of 8-12 weeks were purchased from the Department of Plant Resources, Thapathali, Kathmandu. They were housed in polypropylene cages, in vivarium and maintained at a $12 \mathrm{~h}$ light $-12 \mathrm{~h}$ dark cycle, $24^{\circ} \mathrm{C}-28^{\circ} \mathrm{C}$ temperature, supplied with water and solid pellet food (VRK Nutritional Solutions, Pune, India). All studies involving mice handling were done by strictly following the approved protocol issued by the Ethical Committee of the Central Department of Biotechnology/Tribhuvan University (protocol number 084/2019).

\section{Sperm collection, culture and treatment}

After one week of acclimatization, mice were used for experiments. Male mice were sacrificed by cervical dislocation and dissected with sterile dissection equipment. Testes with attached epididymis were harvested and epididymis were excised and immediately placed in $5 \mathrm{~mL}$ of Dulbecco's Modified Eagle's Media (DMEM) supplemented with $5 \mathrm{mg} / \mathrm{mL}$ bovine serum albumin (BSA), $20 \mathrm{mM}$ hydroxyethyl piperazine ethane sulfonicacid (HEPES) buffer. Epididymis were minced and sperm samples were collected. For all experiments, the final sperm concentration was adjusted to $10^{6}$ cells/ $\mathrm{mL}$. Growth media, supplements and buffers were purchased from HiMedia Laboratories, Mumbai, India.

\section{Sperm isolation, treatment and incubation}

Spermatozoa were incubated in $1 \mathrm{~mL}$ DMEM supplemented with $0.5 \mathrm{mg} / \mathrm{mL}$ BSA and $20 \mathrm{mM}$ HEPES, taken in $1.5 \mathrm{~mL}$ eppendorf tubes. Required volumes of plant extract and medium were added to the tubes making $1 \mathrm{~mL}$ of mixture with extracts concentration equal to $0,25,100,400$, $500,800,1,000$, and 2,000 $\mu \mathrm{g} / \mathrm{mL}$. Vehicle controls were prepared for 1,000 and 2,000 $\mu \mathrm{g} / \mathrm{mL}$ treatments by replacing extract with $80 \%$ ethanol in media. After equilibrating in a $37^{\circ} \mathrm{C}$ incubator, $100 \mu \mathrm{L}$ aliquots of spermatozoa were added to each tube followed by incubation in the incubator for $2 \mathrm{~h}$.

\section{Coomassie and FPNA staining}

Coomassie brilliant blue (G-250) was purchased from Sigma-Aldrich (Baden-Württemberg, Germany) and fluorescein isothiocyanate (FITC) peanut agglutinin (FPNA) from Molecular Probes-Invitrogen (Middlesex County, MA, USA). After $2 \mathrm{~h}$ incubation, $100 \mu \mathrm{L}$ of $40 \%$ formalin was added (final 4\%) to each tube and left at room temperature for $15 \mathrm{~min}$. Centrifugation was done at $500 \times \mathrm{x}$ for 3 minute. The supernatant was aspirated until a loose pellet of $100 \mu \mathrm{L}$ was left at the bottom of the tube. A thin layer of saliva was applied on coverslips and allowed to dry completely. $50 \mu \mathrm{L}$ of sperm suspension was applied on each coverslip and left to air dry. Each coverslips was stained with Coomassie blue for 5 minutes and then washed properly [15]. Finally after complete drying the coverslip were attached on slides with dibutylphthalate polystyrene xylene (DPX) with cells side up. Spermatozoa with acrosome and without acrosome were counted under a bright field microscope under 100X objective.

For FPNA labeling, the tubes were centrifuged at $500 \times \mathrm{xg}$ for 3 minute. The supernatants were aspirated leaving behind $100 \mu \mathrm{L}$ of loose pellets. Thin smear of saliva was applied on coverslips and dried. $50 \mu \mathrm{L}$ of resuspended sperm suspension was added and allowed to settle for 3-5 minutes. Unattached spermatozoa were poured off from the edges and then the coverslips were immersed in $4 \%$ formalin for 30 minutes. After washing with phosphate buffered saline (PBS), the coverslips were treated with $1 \%$ Tween 20 in PBS for 30 minutes. Tween was poured off and $50 \mu \mathrm{L}$ of 250-times diluted FPNA was added followed by incubation at $37^{\circ} \mathrm{C}$ for $1 \mathrm{hr}$ (covered with aluminum foil to avoid light). After removing FPNA, $50 \mu \mathrm{L}$ of ethidium bromide $(\mathrm{EtBr}, 100 \mu \mathrm{g} / \mathrm{mL}$ ) 
was added and incubated for 10 minutes at room temperature in dark. EtBr was removed by washing with PBS and mounted with a drop of $10 \%$ glycerol (in PBS) on a clean slide and the edges sealed with DPX mountant.

Trypan blue staining and hypo-osmotic swelling test

For Trypan blue staining, the eppendorf tubes containing extract treated and control spermatozoa were centrifuged at $500 \times \mathrm{xg}$ for 3 minutes. Supernatant is aspirated and discarded leaving about 20-30 $\mu \mathrm{L}$ of sperm pellet at the bottom. The tubes were immediately kept in ice bucket. From each tube $5 \mu \mathrm{L}$ of sperm suspension was applied on a clean slide and mixed with $5 \mu \mathrm{L}$ of $0.4 \%$ Trypan-blue stain [16]. The mixture was covered with coverslip and observed under 100X objective of a microscope. The percentage of dead spermatozoa (stained blue) was calculated from total 200 counts.

Hypo-osmotic swelling tests [17] were performed for investigating membrane damage of spermatozoa. After incubating with different concentrations of plant extract and control medium for 2 $\mathrm{h}$ at $37^{\circ} \mathrm{C}$, centrifugation was performed for 3 minutes at $700 \times \mathrm{x}$. Pellets of $100 \mu \mathrm{L}$ sample were collected. Sperm suspension in pellet and hypo-osmotic solution were mixed in $1: 10$ ratio, followed by $15 \mathrm{~min}$ incubation at $37^{\circ} \mathrm{C}$. The tubes were centrifuged at $500 \times \mathrm{g}$ for 4 minutes, supernatant discarded leaving around $100 \mu \mathrm{L}$ of loose sperm pellet at the bottom. 1,2 drops of sperm sample was placed on slide, covered by a coverslip. The edges were sealed by DPX and then tail swellings of spermatozoa were observed under microscope [18].

\section{In-vivo fertilization}

After one week acclimatization in vivarium, estrus cycle was checked each day in each female mouse. A direct effect of $A$. vulgaris extract on fertilization was studied by injecting extract into the vagina of estrus females and allowing them to mate with fertile males. Female mice of 6-8 weeks old and male of 8-10 weeks old were used in these experiments.

Experimentations were performed on five sets of mice. For each set of experiment, five mice were used for extract treatment and five as blank control. On the day in which they were found to be in estrus cycle, $10 \mu \mathrm{L}$ of $1,000 \mu \mathrm{g} / \mathrm{mL}$ of Artemisia extract was injected through vagina while for blank control no treatment was done. Immediately, extract injected female mice were caged with male mice in $2: 1$ ratio. Extract injection was performed for three consecutive days in the evening and returned back to the males. In the morning after three days mating, the female mice were checked for the evidence of copulation. Mice with closed copulatory plug and waxy, whitish substances around vaginal area were considered mated. Microscopic examination was performed with the vaginal swab of mated mice for confirming the presence of spermatozoa in their vagina. The female mice were separated and that day was considered day one of pregnancy. Mice were routinely fed throughout the gestation period ( 21 days) and after delivery, number of pups delivered by control mice and extract injected mice were compared [19].

\section{Statistical analysis}

Each experiment was done in triplicate repeats. Microsoft Excel program (MS Office 2013) was used for statistical processing of data, for calculating mean, standard deviations, standard errors, and drawing graphs. Data are expressed as Mean $\pm \mathrm{SD}$. For statistical analysis of the data, two-tailed $t$ test was employed wherever required. $p<0.05$ was considered significant and was calculated from MEDCALC ${ }^{\circledR}$ website [20]. 


\section{RESULTS}

\section{Artemisia leaf extract induces high rate of acrosome reaction}

The nuclei of sperm heads appeared red due to EtBr staining. Spermatozoa labeled with FPNA displayed green, crescent shaped acrosome on the dorsal side of the sperm heads (Fig. 1A). Acrosome reacted spermatozoa lacked such green labeling (Fig. 1B). Acrosomes of mouse spermatozoa were also studied by staining with Coomassie blue. Spermatozoa with intact acrosome displayed crescent-shaped staining on the dorsal surface of head (Fig. 1C, arrow) whereas acrosome reacted spermatozoa lacked such crescent staining (Fig. 1C, arrowhead).

Artemisia leaf extract induced acrosome reaction in spermatozoa. The percentage of acrosome reacted spermatozoa was estimated by counting 200 spermatozoa in the control and treatment samples, labeled with FPNA. In control samples, the percentage of spontaneously acrosome reacted spermatozoa was found to be $22.64 \%$ (Fig. 1D). The proportion of acrosome reacted spermatozoa linearly increased with increasing extract concentration from $25 \mu \mathrm{g} / \mathrm{mL}$ to $1,000 \mu \mathrm{g} / \mathrm{mL}$. The percentage of acrosome reacted spermatozoa further increased at 2,000 $\mu \mathrm{g} / \mathrm{mL}$ extract concentration, but the value was not appreciably higher than that of $1,000 \mu \mathrm{g} / \mathrm{mL}$. Hence the optimum concentration of the extract that induces acrosome reaction in mouse spermatozoa was considered to be $1,000 \mu \mathrm{g} / \mathrm{mL}$. In vehicle control experiements that were done by adding ethanol equivalent to 1,000 $\mu \mathrm{g} / \mathrm{mL}$ and $2,000 \mu \mathrm{g} / \mathrm{mL}$ of extract concentrations, the acrosome reaction rates were not significantly different than that of untreated samples (Fig. 1D). Hence a possibility that ethanol (vehicle)
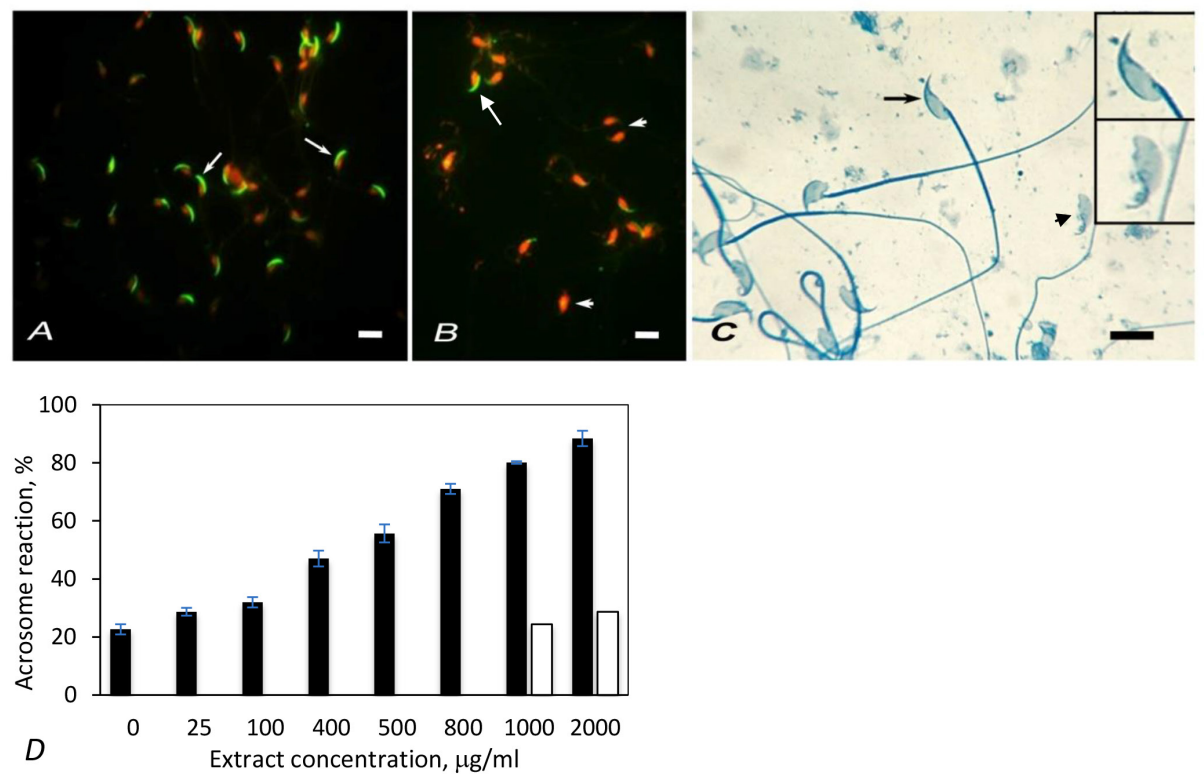

Fig. 1. Effect of Artemisia leaf extract on mouse sperm acrosome. (A) Untreated mouse spermatozoa labeled with fluorescein isothiocyanate (FITC) peanut agglutinin (FPNA) and ethidium bromide (EtBr). Most of spermatozoa displayed crescent shaped acrosomes, labeled with FPNA (green, arrows) on the dorsal surface of nuclei. Nuclei were stained red by EtBr but not clearly visible in the microphograph, because they were overshadowed by bright green fluorescence of FPNA. (B) Mouse spermatozoa treated with 1,000 $\mu \mathrm{g} / \mathrm{mL}$ extract. Few spermatozoa possessed acrosome (green, arrow). Sperm heads are labeled red with $\mathrm{EtBr}$ (arrowheads). (C) Mouse spermatozoa stained with coomassie blue. The acrosome appeared as thick blue line on the dorsal surface of sperm heads (arrow). Thick blue line was absent on acrosome reacted sperm heads (arrowhead). Magnified view of sperm heads with intact and lost acrosome are also shown in the insets. (D) Acrosome reaction of spermatozoa treated with different concentrations of $A$. vulgaris extract. Each plotted point represents a mean value from three experiments. The vertical lines indicate standard deviations. Open columns represent the vehicle controls. Bar in $(A-C), 10 \mu \mathrm{m}$. 
contained in the extracts might cause appreciable of acrosome reaction is ruled out. The effect of extract on acrosome reaction in mouse spermatozoa was also studied by staining spermatozoa with Coomassie blue. This study also showed similar result as that with FPNA staining (Table 1).

\section{Artemisia leaf extract causes viability loss in spermatozoa, but results low rate of membrane damage}

Trypan blue staining is a simple noninvasive rapid technique to determine sperm viability. When stained with Trypan blue, viable spermatozoa with intact cell membrane remain colorless, excluding the stain. Spermatozoa that have damaged membrane (dead) show dark blue staining in the heads due to dye penetration into them (Fig. 2A) [16]. Hence, the percentage of unstained spermatozoa denotes viability.

Mouse spermatozoa treated with hypoosmotic buffer differentiated into two populations - one population with apparently normal looking with straight structure (Fig. 2B) and the other with variously bent neck or tails (Fig. 2C). The latter population comprised live spermatozoa with intact plasma membrane and the former one were dead spermatozoa with damaged plasma membrane.

Incubation with extract caused loss of viability of spermatozoa. The extent of effect was proportional to the concentration of extract (Fig. 2D). Optimum effect was observed at $1,000 \mu \mathrm{g} / \mathrm{mL}$ concentration, as in acrosome reaction. Spermatozoa with Coomassie positive nuclei (dead spermatozoa) observed at this concentration was $55.4 \%$. Treatment with $2,000 \mu \mathrm{g} / \mathrm{mL}$ of extract caused higher viability loss (63.7\%), but the difference in comparision to $1,000 \mu \mathrm{g} / \mathrm{mL}$ was not remarkable in spite of two-folds increase in concentration. Vehicle control experiments were done by incubating spermatozoa with two concentrations of ethanol, equivalent to $1,000-$ and $2,000 \mu \mathrm{g} / \mathrm{mL}$ extract treatments. Ethanol caused slightly higher viability loss $(15.5 ; 17.62 \%)$ than blank controls $(11.71 \%$; Fig. 2D). These observations prove that the viability loss was caused by Artemisia extract, but not due to ethanol.

Treatment with Artemisia leaf extract also caused membrane damage in spermatozoa in a dose dependent manner, but the effect was less conspicuous than viability loss or acrosome damage. At the optimum concentration of extract $(1,000 \mu \mathrm{g} / \mathrm{mL}$ ), membrane damage was only $21.17 \%$ (Fig. $2 \mathrm{E})$.

\section{Artemisia leaf extract reduces fertility in mice}

To study a direct effect on fertility, the extract was injected into the vagina of estrus female mice

Table 1. Acrosome reaction in mouse spermatozoa, induced by various concentrations of Artemisia extract and evaluated by Coomassie staining

\begin{tabular}{lcc}
\hline & Conc of extract $(\mathrm{mg} / \mathrm{mL})$ & Acrosome reaction $(\%)$ \\
\hline 0 & $18.15^{*}$ & 2.62 \\
25 & 22.81 & 2.88 \\
100 & 28.07 & 3.23 \\
400 & 46.39 & 1.59 \\
500 & 54.42 & 1.41 \\
800 & 68.34 & 1.12 \\
1,000 & 78.37 & 1.11 \\
2,000 & 85.91 & 2.56 \\
\hline VC $1,000 \mathrm{mg} / \mathrm{mL}$ & 21.68 & \\
VC $2,000 \mathrm{mg} / \mathrm{mL}$ & 24.1 & \\
\hline
\end{tabular}

*Each value is a mean from three estimations.

VC, vehicle control. 

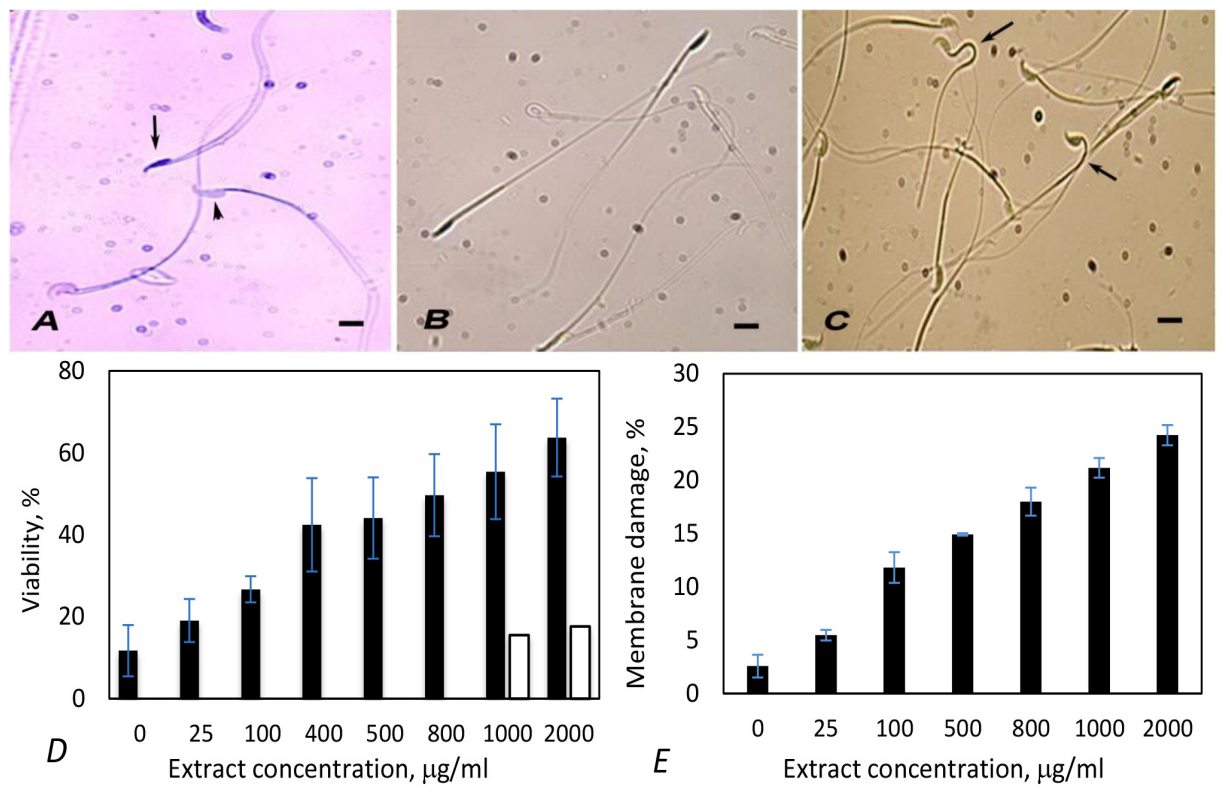

Fig. 2. Effect of Artemisia extract on sperm viability and membrane damage. (A) Trypan blue staining of mouse spermatozoa treated with $1,000 \mu \mathrm{g} / \mathrm{mL}$ extract. Dead spermatozoa showed nuclei stained with Trypan blue (arrow) due to leaky membrane. Live spermatozoa excluded dye pentration, showing unstained or light blue nuclei (arrowhead). (B) Mouse spermatozoa treated with $1,000 \mu \mathrm{g} / \mathrm{mL}$ extract and subjected to hypoosmotic swelling test. Membrane damaged spermatozoa (arrows) were unaffected by hypoosmotic medium and remained straight. (C) Untreated spermatozoa subjected to hypoosmotic swelling test. Live spermatozoa developed various deformations or bendings in the neck and tail regions (arrows) due to the presence of undamaged plasma membrane and endosmotic swelling (see text). (D) Viability of mouse spermatozoa treated with various concentrations of Artemisia leaf extract, evaluated by Trypan blue staining. Open columns represent the vehicle controls. $(E)$ Membrane damage of spermatozoa treated with various concentrations of extract, evaluated by hypoosmotic swelling test. Bar in (A-C), $10 \mu \mathrm{m}$.

and conjugated with males. In the beginning, extract treated and blank control female mice were conjugated for one night and separated. But such mice often failed to be impregnated due to unknown reason. Therefore, the estrus females were intravaginally injected with the extract for three consecutive evenings and kept with males for three nights. By this method the control and treated mice were successfully impregnated and gave birth to healthy pups. The extract caused low fertility in mice. The litter size of the treated mice was nearly half in comparison to the blank control (5.2 verses 9.4, Table 2). The pups developed into healthy and fertile adults some of which were used for breeding or for spermatozoa study. Hence the reduced fertility of Artemisia leaf extract treated mice is not due to ethanol or other stress incurred during the experimental procedure.

\section{DISCUSSION}

In the present work, potential contraceptive effect of Artemisia extract was evaluated by analyzing acrosome reaction, viability loss, membrane damage in mouse spermatozoa and by evaluating fertility loss by intravaginal application. Acrosome plays very important role in fertilization by binding and proteolytically digesting zona pellucida thus helping spermatozoa to enter into oocytes [21]. During normal fertilization process, acrosome intact or recently acrosome reacted spermatozoa bind zona surface through specific ligand-receptor interaction followed by vesiculation and release of acrosomal content. Spermatozoa that have lost acrosome long before encountering zona pellucida cannot perform these two functions hence fail to fertilize. Moreover spermatozoa die shortly after 
Table 2. Effect of Artemisia leaf extract on mouse fertility

\begin{tabular}{ccc}
\hline & \multicolumn{2}{c}{ Number of litters born } \\
\cline { 2 - 3 } Set No. & Control & Treatment \\
\hline 1 & 9 & 0 \\
2 & 10 & 8 \\
3 & 9 & 7 \\
4 & 10 & 5 \\
5 & 9 & 5 \\
\hline Mean \pm SE & $9.4 \pm 0.21^{*}$ & $5 \pm 2.22^{*}$ \\
\hline
\end{tabular}

In treatment mice, $10 \mu \mathrm{L}$ of $1,000 \mu \mathrm{g} / \mathrm{mL}$ extract were intravaginally injected into females consecutively for 3 evenings and kept with males, each night after injection (see text)

${ }^{*} p=0.0137$.

acrosomal exocytosis [22]. The present work has shown that Artemisia leaf extract causes precocious acrosome exocytosis in mouse spermatozoa. The rate of acrosome reaction increased linearly with increase in concentration of the extract, the optimum effect being observed at the concentration $1,000 \mu \mathrm{g} / \mathrm{mL}$. The optimum acrosome reaction observed was $80.4 \%$ which is similar to the optimum effect of Calcium ionophore in mouse spermatozoa and ram spermatozoa [23,24]. Calcium ionophore causes massive influx of $\mathrm{Ca}^{2+}$ into cells and kills them if the treatment is prolonged. Spermatozoa are quickly immobilized by calcium ionophore and hence cannot fertilize oocyte. However they regain hypermobility and resume fertilizing competence if ionophore is promptly removed [25].

FPNA stains the acrosome matrix by binding to glycoconjugates in the acrosome and produces bright green fluorescence under fluorescence microscope. Hence FPNA labeling is a standard method to study presence or absence of acrosome in spermatozoa [26]. Spermatozoa with disrupted acrosome do not label with FPNA (Fig. 1B). FPNA labeling can be used to assess the acrosomal status and the zona-pellucida induced acrosome reaction in stallion [27]. Spermatozoa sample were stained with FPNA, and counterstained with the DNA dye ethidium homodimer. Acrosome-intact stallion spermatozoa were found to display intensively green fluorescence over the acrosomal cap, whereas reacting spermatozoa showed a patchy disrupted image of fluorescence.

The crude extract of $A$. vulgaris at very low concentration, induced high rate of acrosome reaction. The putative substance(s) that induced acrosome reaction might be present in very low proportion in the crude extract. At present, it is not possible to speculate about its chemical nature or molecular pathway of the acrosome reaction. Future investigation should address whether the extract induced acrosome reaction is $\mathrm{Ca}^{2+}$ dependent, whether it affected $\mathrm{Ca}^{2+}$ uptake during capacitation of spermatozoa, whether addition of anion transport inhibitor during extract treatment obstructs $\mathrm{Ca}^{2+}$ uptake [28], or whether it overrides the cAMP/PKA pathway in inducing acrosome reaction [25].

Whereas Artemisia extract induced acrosome reaction at high rate $(80.12 \%$ by $1,000 \mu \mathrm{g} / \mathrm{mL}$ conc) but caused lesser viability loss (55.39\%) and even lower rate of membrane damage (21.17\%). These observations signify that some spermatozoa whose acrosomes have been damaged by the extract are viable and possess intact cell membrane. Quite possibly extract induced acrosome reaction is similar to calcium ionophore induced acrosome reaction in which majority of spermatozoa retain viability $[25,28]$ that can fertilize oocytes when ionophore is removed [25].

In a study carried out by Alvarez et al. [29], ethanol was found to cause loss of acrosome as well as the the equatorial membrane that are needed for gamete fusion. So it was imperative that the effect of ethanol which was used as the sovent (vehicle) of Artemisia extract, be evaluated. Experiments were carried out by replacing extract by $80 \%$ ethanol. Acrosome reacted spermatozoa was 
found to be $21.68 \%$ and $24.1 \%$ when treated with amount of ethanol equivalent to the extract concentration of $1,000 \mu \mathrm{g} / \mathrm{mL}$ and $2,000 \mu \mathrm{g} / \mathrm{mL}$ (vehicle controls) respectively which were slightly higher than the effect of blank control (18.15\%). These observations prove that low concentrations of ethanol used in the present experiment had negligible effect on acrosome reaction.

The extract may exert effect on spermatozoa by generating reactive oxygen species (ROS). As spermatozoan plasma membrane is rich in polyunsaturated fatty acids which can easily undergo lipid peroxidation in the presence of ROS leading to change in fluidity and then degeneration [30]. Therefore, the effect of extract on sperm membrane integrity or viability was analyzed by hypo-osmotic swelling test. Membrane damage increased from $5.47 \%$ in control to $21.17 \%$ on treatment with extract concentration of $1,000 \mu \mathrm{g} / \mathrm{mL}$. These data signify that extract damaged the sperm membrane to some extent but the effect was remarkably less than acrosome damage (Compare Fig. 2E with Fig. 1D) or viability loss (compare with Fig. 2D).

Intravaginal application of Artemisia extract significantly reduced the litter size. Since the extract would directly interact with spermatozoa deposited in vagina after copulation, reduction in litter size would imply that the extract exerts a direct contraceptive effect by interfering in fertilization or implantation. But the treatment resulted only $49 \%$ reduction in litter size. This much effect does not qualify the crude ethanol extract of Artemisia to be used as used as a contraceptive. Nevertheless, there is a scope for further evaluation by treating females with higher dose of the extract since presently injected extract amount $(10 \mu \mathrm{L}$ at $1,000 \mu \mathrm{g} / \mathrm{mL})$ is totally harmless to mice and mice could possibly tolerate higher dose. Furthermore the active contraceptive principle of extract should be purified and screened. To this end, in vitro fertilization techniques would provide more resolution. This technique would allow studying the effect extract on various aspects of fertilization such as sperm-zona binding, zona penetration, sperm-oocyte fusion and early cleavages.

The results provided strong evidence that the extract could be an efficient contraceptive if applied intravaginally. But we have performed tests only on five sets of mice. For irrefutable conclusion, more experiments are needed to be performed with more sets of mice. Moreover several toxicological studies must be performed before it could be tested for human use or veterinary use. In a similar study, hexane fraction of the two plants Achyranthes aspera and Stephania hernandifolia in combination was found to be an effective spermicidal and when applied as a vaginal contraceptive in rats resulted $100 \%$ infertility [31].

\section{REFERENCES}

1. Peachman RR. Weighing the risks and benefits of hormonal contraception. JAMA. 2018;319:1083-4. https://doi.org/10.1001/jama.2018.0448

2. Daniyal M, Akram M. Antifertility activity of medicinal plants. J Chin Med Assoc. 2015;78:382-8. https://doi.org/10.1016/j.jcma.2015.03.008

3. Singh A, Kala S, Kapoor DN. Reversible contraceptive efficacy and safety evaluation of ethanolic extract of Tinospora cordifolia in animal model. Arch Appl Sci Res. 2011;3:587-92.

4. Dehghan MH, Martin T, Dehghanan R. Antifertility effect of Iranian neem seed alcoholic extract on epididymal sperm of mice.J Reprod BioMed. 2005;3:83-9.

5. Pandey AK, Singh P. The Genus Artemisia: a 2012-2017 literature review on chemical composition, antimicrobial, insecticidal and antioxidant activities of essential oils. Medicines. 2017;4:68. https://doi.org/10.3390/medicines4030068

6. Abiri R, Silva ALM, Mesquita LSSD, Mesquita JWCD, Atabaki N, Almeida EBD, et al. Towards a better understanding of Artemisia vulgaris: botany, phytochemistry, pharmacological and biotechnological potential. Food Res Int. 2018;109:403-15. https://doi.org/10.1016/ 
j.foodres.2018.03.072

7. Soon L, Ng PQ, Chellian J, Madheswaran T, Panneerselvam J, Gupta G, et al. Therapeutic potential of Artemisia vulgaris: an insight into underlying immunological mechanisms. J Environ Pathol Toxicol Oncol. 2019;38:205-16. https://doi.org/10.1615/JEnvironPatholToxicolOncol.2019029397

8. Su XZ, Miller LH. The discovery of artemisinin and the nobel prize in physiology or medicine. Sci China Life Sci. 2015;58:1175-9. https://doi.org/10.1007/s11427-015-4948-7

9. Malik S, de Mesquita LSS, Silva CR, de Mesquita JWC, Rocha EDS, Bose J, et al. Chemical profile and biological activities of essential oil from Artemisia vulgaris L. cultivated in Brazil. Pharmaceuticals. 2019;12:49. https://doi.org/10.3390/ph12020049

10. Satyal P, Paudel P, Kafle A, Pokharel SK, Lamichhane B, Dosoky NS, et al. Bioactivities of volatile components from Nepalese artemisia species. Nat Prod Commun. 2012;7:1651-8. https:// doi.org/10.1177/1934578X1200701228

11. Shaik A, Kanhere RS, Cuddapah R, Nelson KS, Vara PR, Sibyala S. Antifertility activity of Artemisia vulgaris leaves on female wistar rats. Chin J Nat Med. 2014;12:180-5. https://doi. org/10.1016/S1875-5364(14)60030-3

12. Pal M, Pandey SK. Effect of Artemisia vulgaris isolated extract and determination of AD50 and LD50 for antifertility and mortality of Callosobruchus chinensis (L.).J Ecophysiol Occup Hlth. 2011;11:101-6.

13. Narwaria A, Khosa RL, Dhar SK. Experimental studies on Artemisia vulgaris: a possible antifertility drug. Anc Sci Life. 1994;14:10-5.

14. Abolaji AO, Eteng MU, Ebong PE, Brisibe EA, Dar A, Kabir N, et al. A safety assessment of the antimalarial herb Artemisia annua during pregnancy in Wistar rats. Phytother Res. 2013;27:647-54. https://doi.org/10.1002/ptr.4760

15. Larson JL, Miller DJ. Simple histochemical stain for acrosomes on sperm from several species. Mol Reprod Dev. 1999;52:445-9. https://doi.org/10.1002/(SICI)10982795(199904)52:4<445::AID-MRD14>3.0.CO;2-6

16. Strober W. Trypan blue exclusion test of cell viability. Curr Protoc Immunol. 1997;111:A3. B.1-A3.B.3. https://doi.org/10.1002/0471142735.ima03bs111

17. World Health Organization [WHO]. WHO laboratory manual for the examination and processing of human semen. 5th ed. Geneva, Switzerland:WHO; 2010.

18. Jeyendran RS, Ven HHVD, Perez-Pelaez M, Crabo BG, Zaneveld LJD. Development of an assay to assess the functional integrity of the human sperm membrane and its relationship to other semen characteristics. Reproduction. 1984;70:219-28. https://doi.org/10.1530/ jrf.0.0700219

19. Naz RK. Can curcumin provide an ideal contraceptive? Mol Reprod Dev. 2011;78:116-23. https://doi.org/10.1002/mrd.21276

20. MEDCALC. Free statistical calculators [Internet]. [2020 Aug 8]. https://www.medcalc.org/ calc/comparison_of_means.php?fbclid=IwAR3UoPsut1u1k38yCOqw Ap1mheuxr BIkaZnGY4Q24zv-cVCOgGohrY_kwQ.

21. Yanagimachi R. Fertility of mammalian spermatozoa: its development and relativity. Zygote. 1994;2:371-2. https://doi.org/10.1017/S0967199400002240

22. Tzvetkova P, Zhu WJ, Li J, Tzvetkov D. Evaluation on sperm acrosome integrity of infertile men with varicocele. J Reprodd Contracep. 2007;18:19-24. https://doi.org/10.1016/S1001$7844(07) 60003-0$

23. El-Shahat KH, Taysser MI, Badr MR, Zaki, KA. Effect of heparin, caffeine and calcium ionophore A23187 on in vitro induction of the acrosome reaction of fresh ram spermatozoa. Asian 
Pac J Reprod 2016;5:148-55. https://doi.org/10.1016/j.apjr.2016.01.012

24. Manandhar G, Toshimori K. Exposure of sperm head equatorin after acrosome reaction and its fate after fertilization in mice. Biol Reprod. 2001;65:1425-36. https://doi.org/10.1095/biolreprod65.5.1425

25. Tateno H, Krapf D, Hino T, Sánchez-Cárdenas C, Darszon A, Yanagimachi R, et al. Ca ${ }^{2+}$ ionophore A23187 can make mouse spermatozoa capable of fertilizing in vitro without activation of cAMP-dependent phosphorylation pathways. Proc Natl Acad Sci USA. 2013;110:18543-8. https://doi.org/10.1073/pnas.1317113110

26. Manandhar G, Miranda-Vizuete A, Pedrajas JR., Krause WJ, Zimmerman S, Sutovsky M, et al. Peroxiredoxin 2 and peroxidase enzymatic activity of mammalian spermatozoa. Biol Reprod. 2009;80:1168-77. https://doi.org/10.1095/biolreprod.108.071738

27. Cheng FP, Fazeli A, Voorthout WF, Marks A, Bevers MM, Colenbrander B. Use of peanut agglutinin to access the acrosomal status and the zona pellucida-induced acrosome reaction in stallion spermatozoa. J Androl. 1996;17:674-82. https://doi.org/10.1002/j.1939-4640.1996. tb01852.x

28. Ruknudin A, Silver IA. Ca2+ uptake during capacitation of mouse spermatozoa and the effect of an anion transport inhibitor on $\mathrm{Ca}^{2+}$ uptake. Mol Reprod Dev. 1990;26:63-8. https://doi. org/10.1002/mrd.1080260110

29. Alvarez JG, Lee MA, Iiozzo RV, Lopez I, Touchstone JC, Storey BT. Ethanol accelerates acrosomal loss in human spermatozoa. J Androl. 1988;9:357-66. https://doi.org/10.1002/j.19394640.1988.tb01065.x

30. Thiangtum K, Pinyopummin A, Hori T, Kawakami E, Tsutsui T. Effect of catalase and superoxide dismutase on motility, viability and acrosomal integrity of frozen-thawed cat spermatozoa. Reprod Domest Anim. 2009;44:369-72. https://doi.org/10.1111/j.1439-0531.2009.01420.x

31. Paul D, De D, Ali KM, Chatterjee K, Nandi DK, Ghosh D. Comparative study on the spermicidal activity of organic solvent fractions from hydroethanolic extracts of Achyranthes aspera and Stephania hernandifolia in human and rat sperm. Contraception. 2010;81:355-61. https:// doi.org/10.1016/j.contraception.2009.09.001 